UCRL-ID-119140

\title{
EQ3/6 Software Test and Verification Report 9/94
}

\section{Tad Kishi}

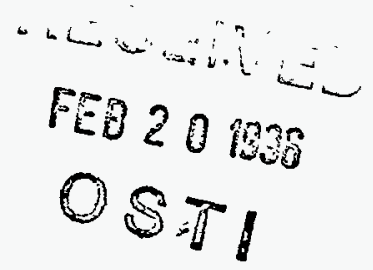

Date Written: September 1994

Date Published: February 1996

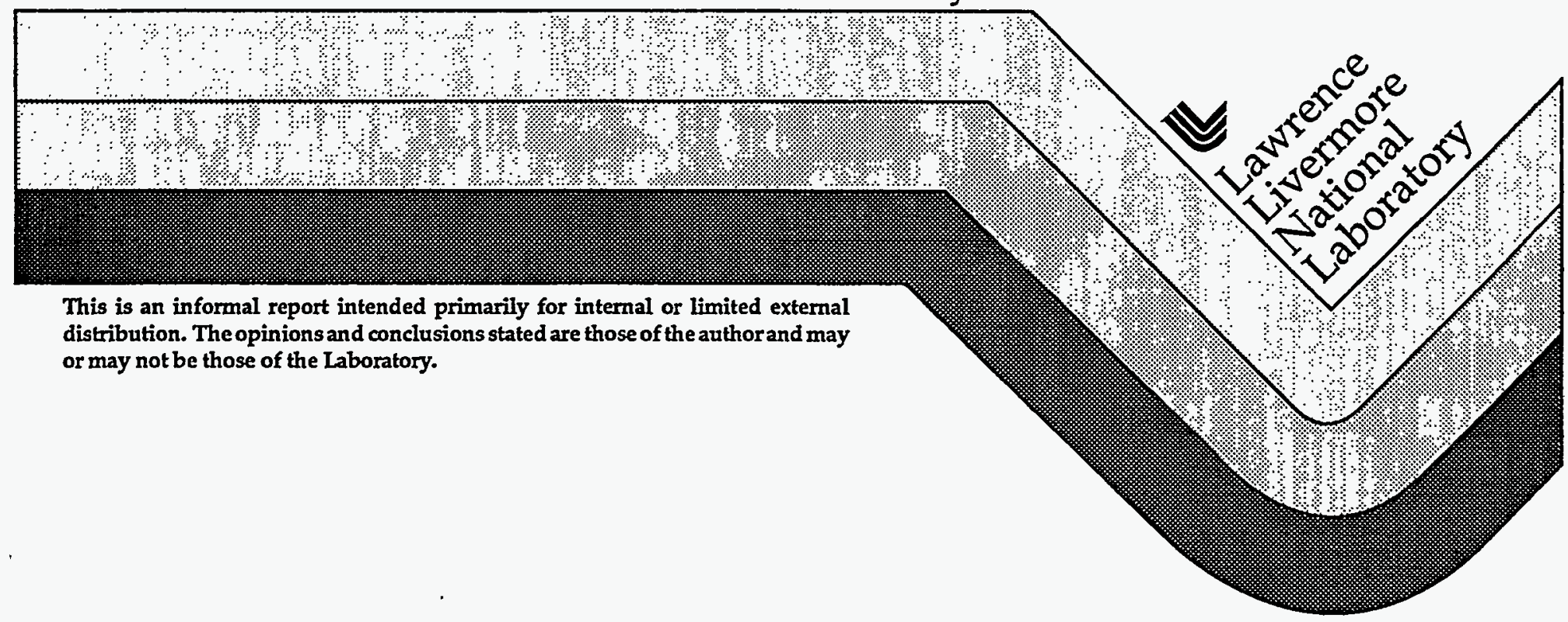




\section{DISCLAIMER}

This document was prepared as an account of work sponsored by an agency of the United States Government. Neither the United States Government nor the University of California nor any of their employees, makes any warranty, express or implied, or assumes any legal liability or responsibility for the accuracy, completeness, or usefulness of any information, apparatus, product, or process disclosed, or represents that its use would not infringe privately owned rights. Reference herein to any specific commercial products, process, or service by trade name, trademark, manufacturer, or otherwise, does not necessarily constitute or imply its endorsement, recommendation, or favoring by the United States Government or the University of California. The views and opinions of authors expressed herein do not necessarily state or reflect those of the United States Government or the University of California, and shall not be used for advertising or product endorsement purposes.

This report has been reproduced directly from the best available copy.

Available to $D O E$ and $D O E$ contractors from the

Office of Scientific and Technical Information

P.O. Box 62, Oak Ridge, TN 37831

Prices available from (615) 576-8401

Available to the public from the

National Technical Information Service

U.S. Department of Commerce

5285 Port Royal Road

Springfield, VA 22161

Prepared by Yucca Mountain Site Characterization Project (MMP) participants as part of the Civilian Radioactive Waste Management Program. The YMP is managed by the Yucca Mountain Site Characterization Project Office of the U.S. Department of Energy, Las Vegas, Nevada. 


\section{EQ3/6 Software Test and Verification Report 9/94 Tad Kishi}

This document is the Software Test and Verification Report (STVR) for the EQ3/6 suite of codes as stipulated in the Individual Software Plan for Initial Qualification of EQ3/6 (ISP. NF-07, Revision 1, 11/25/92). The "Test and Verification" (i.e., "V and V" in the language of QP 3.2, Revision 2, June 21, 1994 which is now the operative controlling procedure) activity has been completed as of this date, September, 8, 1994. The Table of Content lists the items covered in this report. Files listed in Appendix B, C, and D are not included in this report because of the volume information is much too large. These documents will be available at the Records Information Center (RIS). 

EQ3/6 Software Test and Verification Report 9/94

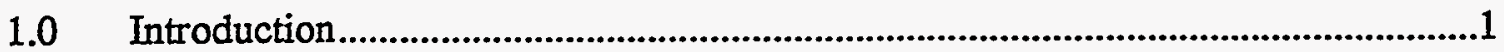

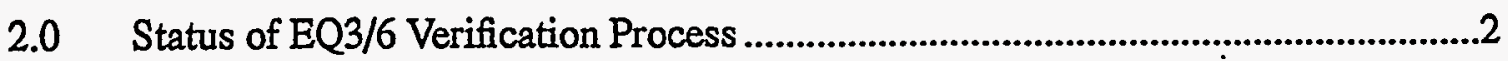

2.1 ISP-NF-07 Revision 1 Applicable Items .....................................................2

2.1.1 Project Management .........................................................................2

2.1.2 Configuration Management (CM) ...................................................2

2.1.3 Software Test and Verification Report (STVR) ................................3

2.1.4 Software Test and Verification Plan (STVP) ....................................3

$2.2 \quad$ EQPT...............................................................................................

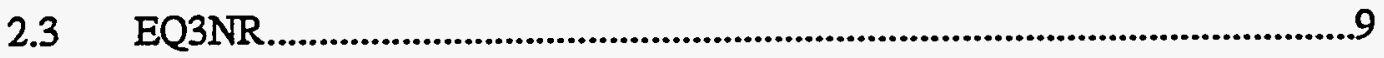

2.3.1 Pre-Newton-Raphson ................................................................................10

2.3.2 Newton-Raphson Method ..................................................................12

2.3.3 Post Newton-Raphson Procedures.................................................14

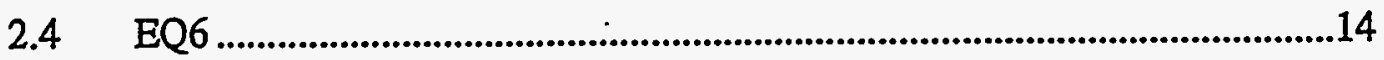

3.0 Comments on the Verification Process ..............................................................15

3.1 Meeting Requirements ...........................................................................15

3.2 Applying the Software Test and Verification Plan (STVP) ........................16

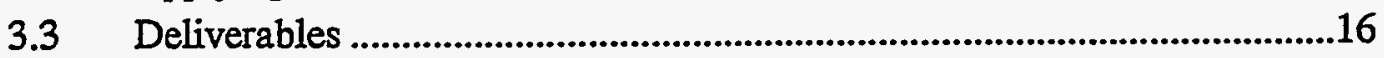

3.3.1 Electronic Notebook .........................................................................17

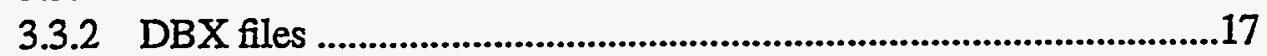

3.3.3 "Reverse Engineered" files ............................................................17

3.3.4 Flint output files ..............................................................................17

3.3.5 Summary Matrix .................................................................................17

$4.0 \quad$ References ..................................................................................................19

Appendix A (ZNOTEBK.DOC)....................................................................................22

Appendix B ("Reverse Engineered" filenames *.DOC).....................................................26

Appendix C (DBX output files *.outx) ...........................................................................29

Appendix D (FLINT *.xrf filenames) ...........................................................................32

Appendix E Acronyms ........................................................................................................33 


\section{.}




\title{
EQ3/6 Software Test and Verification Report 8 /94
}

\author{
Tad Kishi
}

\subsection{Introduction}

This document is the Software Test and Verification Report (STVR) for the EQ3/6 suite of codes as stipulated in the Individual Software Plan for Initial Qualification of EQ3/6 (ISP-NF-07, Revision $1,11 / 25 / 92)^{15}$. The software codes, EQPT, EQ3NR, EQ6, and the software library EQLIB constitute the EQ3/6 software package. This software test and verification project for EQ3/6 was started under the requirements of the LLNL Yucca Mountain Project Software Quality Assurance Plan (SQAP), Revision 0, December 14, 1989, but QP 3.2, Revision 2, June 21, 1994 is now the operative controlling procedure. This is a "V and V" report in the language of QP 3.2, Revision 2 . Since EQ3/6 software category is "Existing Large Scientific and Engineering Software" as defined in the SQAP ${ }^{17}$, the applicable requirements to be tested and verified against are found in the EQ3/6, Version 7 documents (UCRL-MA-110661 PT I - IV) 4,26,27,28,29. It was, however, necessary to consult other technical documents $1,2,3,7,10,16,19,24,25$ in order to meet additional requirements (i.e., pertaining to "standard" computational procedures) lacking in the EQ3/6, Version 7 documents. Because the author of this report does not have a background in geochemistry, other technical sources ${ }^{11,12,14,20}$ were consulted in order to acquire some familiarity wwith geochemistry $^{20}$, the terminology involved, and to review comparable computational methods ${ }^{-11,12,14,21}$, especially,. geochemical aqueous speciation-solubility calculations.

The software for the EQ3/6 package consists of approximately 47,000 lines of FORTRAN77 source code and runs on UNIX platforms ranging from workstations to supercomputers. The physical control of EQ3/6 software package and documentation is on a SUN SPARC station. ISP-NF-06 describes the Configuration Management requirements for maintenance of EQ3/6. The initial software package EQ3/6 Version 7.1 was received from the Configuration Management (CM) system on August 28, 1992 (See 2.1.2). For this V and V project, the software package EQ3/6 Version 7.1 was treated as "acquired code." On August 11, 1993, EQ3/6 Version 7.2 replaced EQ3/6 Version 7.1 because of programming changes (See ZNOTEBK.DOC in Appendix A). On January 12, 1994, EQ3/6 Version 7.2a replaced EQ3/6 Version 7.2 and the test and verification of EQ6 started at this point. Minor bugs found in EQ3/6 Version 7.2 during the test and verification of EQ6 will be corrected in Version $7.2 \mathrm{~b}$.

Walkthroughs of each principal software packages, EQPT, EQ3NR, and EQ6 were conducted in order to understand the computational procedures involved, to determine any commonality in procedures, and then to establish a plan for the test and verification of EQ3/6. It became evident that all three phases depended upon solving an $\mathrm{n} \times \mathrm{n}$ matrix by the Newton-Raphson Method. Thus, a great deal of emphasis on the test and verification of this procedure was carried out on the first code in the software package EQPT (See Section 2.2). Members of the EQ3/6 test case library were used in this work. While the V \& V procedure is described in Section 2.1.4, a special feature of the UNIX $\mathrm{DBX}^{5}$ was used in order to obtain hard copy output and to provide reproducibility of the DBX runs. 
Additionally, in order to easily rewrite equations (see "Reverse Engineering" Section 2.1.4) consistent with those in the EQ3/6 documents, the software publisher on the Sun workstation,

Framemaker ${ }^{9}$, was used for this activity.

\subsection{Status of EQ3/6 Verification Process}

\subsection{ISP-NF-07 Revision 1 Applicable Items}

\subsubsection{Project Management}

Because the EQ3/6 software package is very large, the software tool FORTRAN-LINT (FLINT) ${ }^{8}$, a licensed software accessible on the LLNL Information Technology Center Sun platform, was used exclusively in order to obtain cross-reference file (filename.xrf) and the call-tree (filename.tre) of listings of each source file. The output files, *.xrf and *.tre, were then transported over the unclassified network to the Sun SPARC station S64. The *.tre output is equivalent to a flow diagram of main and its calls to subprograms. This flow diagram allowed a systematic approach to the V \& V process. Another licensed software, Framemaker ${ }^{9}$ was used in order to write equations during the "Reverse Engineering (see 2.1.4)" activity that would be consistent with those in the EQ3/6 documents.

\subsubsection{Configuration Management (CM)}

The deliverables and software were originally controlled in accordance with TIP-YM-11, which is now replaced by QP 3.2, Rev. 2. The physical control of EQ3/6 software package and documentation was on a SUN workstation (S45). ISP-NF-06 describes the Configuration Management requirements for maintenance of EQ3/6. Thus, CM of the software is not the responsibility of the author of this report. During the verification process, two types of errors have been encountered: software and document. When a possible error is encountered, the issue becomes: was the program correct and the document incorrect or was the document correct and the coding incorrect? If the error was programming, the error was recorded in the electronic notebook and both the Task Leader (TL) and the code author were notified of this event. If a change in the software is quality affecting, a new source code was officially obtained from the $\mathrm{CM}$ system and the process of reverification continued from this point. Errors in the EQ3/6 documents must also be resolved when the computational procedure of a program differs from the document. Of course, there is the possibility that the software is consistent with the document and both are in error: but that possibility of model error is not the issue here.

The initial software package EQ3/6 Version 7.1 was received from the Configuration Management (CM) system on August 28, 1992 (See 2.1.2). For this V and V project, the software package EQ3/ 6 Version 7.1 was treated as "acquired code." On August 11, 1993, EQ3/6 Version 7.2 replaced EQ3/6 Version 7.1 because of programming changes (See ZNOTEBK.DOC in Appendix A). On January $12,1994, E Q 3 / 6$ Version 7.2a replaced EQ3/6 Version 7.2 and the test and verification of EQ6 started at this point. Minor bugs found in EQ3/6 Version 7.2 during the test and verification of EQ6 will be corrected in Version 7.2b. 
The events leading to changes in the EQ3/6 software package have been recorded in the electronic notebook, ZNOTEBOOK, which is included in this report as appendix A. It should be noted that the author of this report did not alter any modules during the $\mathrm{V} \& \mathrm{~V}$ activities.

\subsubsection{Software Test and Verification Report (STVR)}

This report is the official document that reports the status of EQ3/6 "V \& V" project.

\subsubsection{Software Test and Verification Plan (STVP ${ }^{23}$ )}

The procedures set forth in the STVP ${ }^{23}$ (Appendix, ISP-NF-07, Revision 1) have been carried out for the four principal components (EQPT, EQ3NR, EQ6, \& EQLIB-library modules) in the EQ3/6 software package. $\mathrm{DBX}$, the UNIX debugging program, has been used for isolating and changing data while running a program. However, for unit testing of a computational procedure, another method was used in place of DBX. The term "Reverse Engineering" best describes this procedure. The steps include examining a given computational procedure in the source code, "reverse engineer" the equation(s) for a given procedure, and compare the result(s) against information (i.e., requirements) in the EQ3/6, Version 7.0 documents.

At first, notes and equations were hand written onto the particular source code listings. It soon became apparent that, as time progressed, these notes and equations were not only undecipherable, but also impossible to keep up-to-date. Even writing symbols consistent with the documents became an impossible task. A decision was made to abandon the handwritten approach and, in its place, use FrameMaker ${ }^{9}$ (Frame Technology Corp. publishing software) as my means of documenting the verification process. The process is as follows: import the source code into a FrameMaker document, delete any extraneous lines, and write the equations into the particular line of the source code with symbols cut and pasted from the official EQ3/6 documents. By doing so, errors in coding and document errors have been easier to identify and compare.

The above method was employed on all mathematical subroutines for EQ3/6 and does not replace the requirement of "walkthroughs" for each module. In fact, it is a method used in addition to other tools and methods listed in the STVP ${ }^{23}$ during the "walkthroughs." However, the use of the "Reverse Engineering" technique on a simple routine, such as tlg(x) (i.e., calculate the $\log$ of $x$ ), during the "walkthroughs" is obviously unnecessary. Furthermore, all input variables and local variables are examined in detail in order to determine whether they are properly set (i.e., appear as lefthand members in equations) before using them in a particular module.

\subsection{EQPT}

EQPT is a data file preprocessor for the EQ3/6 software package. EQPT processes a set of formatted ASCII files named data 0 with suffixes com, sup, nea, hmw, and pit and produces a corresponding unformatted data file named data1 and also a formatted datalf file. To run EQ3NR or EQ6, the user must select which of the five output data files is most appropriate for a given problem. EQPT, as well as EQ3NR and EQ6, is supported by a set of subroutines in the library file EQLIB. These 
subroutines from EQLIB were verified along with the above three software units rather than verifying them separately. $\mathrm{DBX}{ }^{5}$ was used extensively in verifying EQPT. Since EQPT is a database software package, all of the $\mathrm{I} / \mathrm{O}$ subroutines and system interface routines were reviewed and verified. The focus, however, was on the subroutine msolvr.f, which solves an $\mathrm{n} \times \mathrm{n}$ matrix. The numerical technique applied is $\mathrm{L} / \mathrm{U}$ decomposition with partial pivoting. This procedure is also key to the Newton-Raphson method used in EQ3NR and EQ6. In the sequence, eqpt.f-wrpar.f-intrp.f-Isqp.fpolx.f (see Diagram 1 or EQPT.tre), the subroutine msolvr.f is called by polx.f. The program msolvr.f and its supporting subroutines are based on the versions extracted from a public domain software library either called LINPACK ${ }^{6}$ or EISPACK.

Initially, DBX runs were made with the data0 file, data0.com.R16. Parameters read in from the data0 file corresponding to a standard temperature grid are scaled and fitted to a polynomial by means of a least squares fit for two temperature ranges. The module msolvr.f calculates the interpolating polynomial coefficients that are written to the datal file instead of the original input values. While msolvr.f and the rest of the mathematical software programs have been used by scientists for many years, the equivalent of a detailed "requirements document" does not exist for any of the modules to compare against. Futhermore, the data0 file used in this effort was inadequate for the verification process.

Although the functional requirements for the modules in LINPACK are well known, though perhaps the design details may not be, the detailed information necessary for this V \& V was not readily available. Thus, a number of technical papers ${ }^{3,6,10,25}$ and text books ${ }^{1,2.7,16,19,24}$ were reviewed in order to understand the algorithm used by msolvr.f and to find sample problems to test the modules. The module msolvr.f calls: 1) sgeco.f that factors a real matrix by Gaussian elimination with partial pivoting and estimates the "condition" number of the matrix; 2) sgesl.f that solves the real matrix, $\mathbf{A} \mathbf{x}=\mathbf{b}$; and 3 ) itrefn.f that computes residuals and uses these to obtain a NewtonRaphson correction to the $\mathbf{x}$ vector. Documents that are relevant to these modules are:

1) SIMSOLVR.DOC or MSOLVR.DOC

Listing of a DBX run that stops in "msolvr.f": 64 and data loads a special test data ( $4 \times 4$ matrix), computes the reciprocal of the condition number (rcond) and solves the matrix equation, and then forces an iterative procedure (i.e., itrefn.f) to compute residuals.

The following are the test and verification comments:

* This is a DBX run with test data after

*\$ entering msolvr.f:64. The test data are

* $\$$ L/U decomposed by sgeco.f with partial

* pivoting and then solved by sgesl.f.

* \$ In order to force an iterative procedure

* $\$$ on the test data, the reciprocal of the

* $\$$ condition number (rcond) is changed to

$* \$ 0.1 *$ eps 100 such that the test:

*\$ msolvr.f:105: if(rcond.le.eps100) is .true. and the

*\$ subroutine itrefn.f is called. The

* $\$$ fudged solution converges with two

* $\$$ iterations and agrees within error limits (i.e., $100 *$ machine eps).

*\$ See the test matrix aa[ below.

* The subrt msolvr.f does very little except

$* \$$ to call sgeco (thus sgefa.f) and sgesl.f. 
$*$ As noted, an iterative solution calling

* $\$$ itrefn.f is initiated by testing rcond.

$* \$$ No further verification of msolvr.f are

*\$ necessary.

*\$ T. Kishi December 3, 1992

\section{2) RCOND.DOC}

The document contains two parts: "Reverse Engineering" the equations used in rcond.f, and a listing of a DBX run on rcond.f that

loads data into a new matrix in order to compute "rcond" and compares it with the standard definition.

To test for singularity, rcond, the reciprocal of the condition number is calculated in the subroutine sgeco.f. However, the test rfondseps, is not sufficient to guarantee singularity. The condition number of the matrix $A=\left|A^{-1}\right| A \mid$ with respect to the norms of $A$ and $A^{-1}$. Since the inverse $A^{-1}$ is not calculated, an estimate of rcond is calculated in the subroutine sgeco.f. The following lines of comment are listed in sgeco.f: the estimate of $\left\|A^{-1}|=| z|\div| y\right\|$ where $A z=y$ and $A^{\dagger} y=e$ (transpose $\mathrm{A}=A^{\dagger}$ ). The components of e are chosen to cause maximum local growth in the elements of $w$ where $U^{\dagger} w=e$. The vectors are frequently rescaled to avoid overflow.

Solving the system,

$$
A x=b,
$$

let $\hat{x}$ be an approximate solution and $\mathbf{r}$ the residual.

Subtracting gives

$$
A \hat{x}=\boldsymbol{b}-\boldsymbol{r}
$$

Since $e=x-\hat{x}$,

$$
A(x-\hat{x})=r
$$

Now

$$
e=A^{-1} r \text {. }
$$

and

$$
|b| \leq|A\|\cdot\| x|
$$

$$
|e| \leq\left\|A^{-1}\right\| \cdot \mid r \|
$$

Rearranging terms and define a new condition number,

$$
\operatorname{rcond}=\frac{1}{\operatorname{cond}(A))} \leq\left(\frac{\|r\|}{\|b\|}\right) /\left(\frac{\|e\|}{\|x\|}\right) \text {. }
$$

In estimating $\left\|A^{-1}\right\|$,

$$
\begin{aligned}
& e=A^{-1} z \text { and }\|e\| \leq\left|A^{-1}\right| \cdot\|z\| . \\
& \left\|A^{-1}\right\| \geq \frac{\|z\|_{\infty}}{\|e\|_{\infty}}
\end{aligned}
$$

The idea is to choose e such that $\mid e \|_{\infty}=1$ (i.e., $e_{i}= \pm 1$ ), such that $\|z\|_{\infty}$ becomes as large as possible. 
In the code sgeco.f, we consider the upper triangular matrix $U$. The backward substitution for the solution of $U z=e$ is solved by

The summation process $\left(\sum_{k}=\left(\sum_{i=k+1}^{n} u_{k i} z_{i}\right)\right.$ is equivalent to the procedure

for $\mathrm{i}=1$ to $\mathrm{k}-1$ do

$p_{i}=p_{i}+u_{i k} z_{k}$ and choose $p_{i}$ such that,

$\left(e_{k}-z_{k}\right)^{\prime} u_{k k}$ assumes the largest possible value.

The variable $e_{k}=\left(\operatorname{sign} z_{k} 1.0\right)$. That is, $e_{k}= \pm 1.0$ depending upon which of $\left(1-z_{k}\right)^{\prime} u_{k k}$ and $\left(-1-z_{k}\right)^{/ u_{k k}}$ is largest.

The sequence of mathematical steps that arrive at calculating rcond is listed in the document RCOND.DOC and a detailed examination of the steps is listed in a series of DBX runs

A second part of this report takes a sample problem from J. Maron, data loads the matrix, computes the value rcond by sgeco.f and then compares the result with the mathematical definition of rcond.

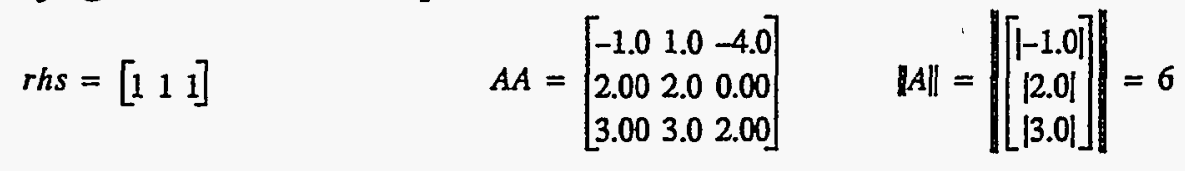

$\operatorname{Det}(\mathrm{A})=4$

$$
A^{-1}=\left[\begin{array}{ccc}
2.00 & 7.0 & -4.0 \\
2.00 & -5.0 & 4.0 \\
0.00 & -3.0 & 2.0
\end{array}\right] / 4 \quad\left\|A^{-1}\right\|=\frac{1}{4} \times\left\|\left[\begin{array}{c}
7.00 \\
1-5.0 \\
-3.0
\end{array}\right]\right\|=15 / 4
$$

cond $A=\|A\| A^{-1} \|=6(15 / 4)$

“ cond $A=6 *(15 / 4)=22.5$

rcond $=1 /$ cond $A=1 / 22.5=0.04444444444$

rcond computed by sgeco.f:

rcond $=$ ynorm/anorm $=0.340175953079178917 / 6.0=0.0566959921798631528$

Thus, rcond hand calculated from the sample problem using the mathematical definition and compared with rcond from sgeco.f both satisfy: rcond $\leq 1.0$.

The second part of this document also answers the following:

Does $\boldsymbol{L U}=\boldsymbol{p A}$, where $\mathrm{p}$ is the permutation vector and interpret $-\boldsymbol{M}=\boldsymbol{L}$. ?

That is, $M$, the multipliers, actually appear as the elements of the lower triangular matrix.

Using the $\mathrm{DBX}$ output and hand calculations, the original $\mathrm{A}[\mathrm{m}$ matrix with row interchanges does indeed satisfy $L[* U[]=A[]$. 


\section{3) SGEFA.DOC}

This document lists two parts: i) Gaussian elimination of a sample 4X4 matrix and compares sgefa.f against hand calculated values; ii) shows that $\mathbf{P A}=\mathbf{L U}$ by following the sequence of calls from wrpar.f (intrp.f,Isqp.f, polx.f, msolvr.f, sgeco.f,.), where $P$ is the permutation matrix (i.e., formed from partial pivoting) and $\mathrm{L}$ and $\mathrm{U}$ are the lower and upper triangular matrices formed by Gaussian

Given elimination.

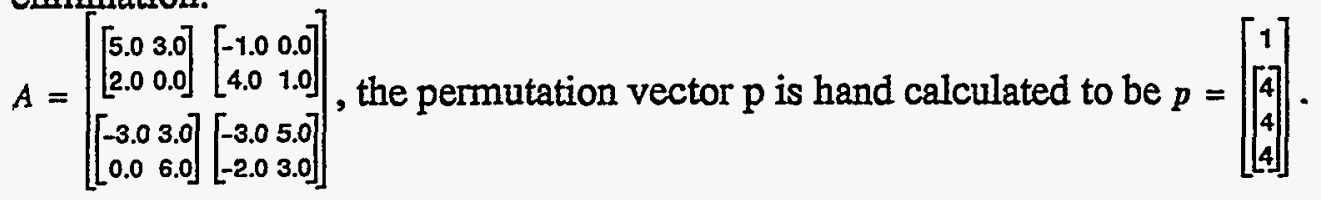

The following is the hand calculated $L U$ decomposition of $A$

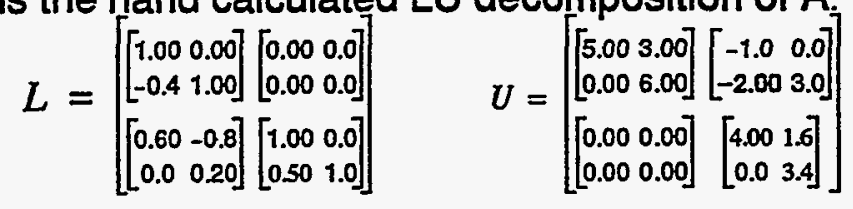

The corresponding values from sgefa.f:

"at end of sgefa: 136 " = "at end of sgefa:136"

("sgefa'sgefa'ipvt) $[1]=1,[2]=4,[3]=4,[4]=4$

( ${ }^{\star}$ 'sgefa'sgefa'a) $[1,1]=5.0,[2,1]=-0.400000000000000022,[3,1]=0.600000000000000089,[4,1]=-0.0$

("'sgefa'sgefa'a) $[1,2]=3.0,[2,2]=6.0,[3,2]=-0.800000000000000044,[4,2]=0.200000000000000011$

(*'sgefa'sgefa'a) $[1,3]=-1.0,[2,3]=-2.0,3,3]=4.0,[4,3]=0.5$

("sgefa'sgefa'a) $[1,4]=0.0,[2,4]=3.0,[3,4]=1.60000000000000009,[4,4]=3.39999999999999947$

The values from sgefa.f agree with the hand calculations to at least 17 significant digits.

4) ITREFN.DOC .

This document is an edited version of a DBX run on the subroutine itrefn.f that includes a data loading of a sample problem and a doctored solution vector to test convergence.

Note: A script for this DBX run is listed from top of page 4 to line 10 of page 10.

The following output is from subroutine itrefn.f that computes residuals, using a partial product algorithm, and iteratively refines the solution vector.

We are to solve $A x=b$. The following technical information is abstracted from Wilkinson's "The Solution of $\mathrm{Ml}$-Conditioned Linear Equations":

$$
A x=b
$$

Let $r^{(s)}$ represent the s-th residual vector corresponding to the $s$-th iteration.

$$
r^{(s)}=b-A x^{(s)}
$$

If $\mathrm{e}$ is the exact solution for 1 -st iterative solution of

$$
A x=r^{(1)}
$$

then

$$
A\left(x^{(1)}+e\right)=b \text {. }
$$

Since the exact $e$ is not attained, we continue a sequence of approximations using residuals $r^{(s)}$, cor- 
rections $e^{(s)}$ and solutions $x^{(s)}$.

The relative errors for floating point operations are of the order

$$
\left(\left|\varepsilon_{i}\right| \leq 2^{-t}\right) \text { where the mantissa contains } t \text { binary digits. }
$$

Wilkinson shows that with the use of accumulating inner products if the first solution $x^{(1)}$ has some correct figures, say $p$ digits $(p>1)$, then $x^{(2)}$ will have $2 p$ (or more) correct figures compared with $p$ (or more) correct figures in $x^{(1)}$. However, the residual does not necessarily diminish. By repetitive iterations

$$
\left\|e^{(s)}\right\|_{\infty} \leq 2^{-t}\left\|x{ }^{(s)}\right\|_{\infty}
$$

or the relative error is less than one part in $2^{t}$.

The FORTRAN statements extracted from itrefn.f (lines $201, \ldots$ ) list a procedure which retain double precision accuracy for the accumulation of inner products in computing residuals. This is in keeping with Wilkinson's statement, "Our analysis shows that the accumulation of inner products is absolutely vital, and if this cannot conveniently be done, the true double precision must be used." We note that in the code the data const $=2 * 23=8388608$. This constant is approximately $10^{7}$.

$* * * * * * * * * * * * * * * * * * * * * * * * * * * * * * * * * * * * * * * * *$

*\$ Test \& Verification Comments

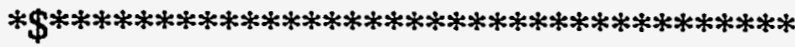

$* \$$

* $\$$ For data0=data0.com.R16 subroutine itrefn would normally be bypassed since subroutine msolvr tests rcond <=eps100 (which is .true.) on linemsolvr.f:105 and terminates. In .order to force entry to itrefn.f, test data of a sample problem is loaded on entry to msolvr:64, at line msolvr:105, rcond $>=$ eps 100 is set false, and the solution vector del[ is also changed. Lines 23-61 of this DBX input reflect the changes. The doctored solution vector converges to the correct values with two iterations (except for round-off errors). Itrefn.f splits the solution vector $x[$ into two parts by means of a constant, const $=2 * 23$. The $\log \_$ten $(23)$ is approximately 8 eight significant digits(i.e., real $\left.* 8\right)$. Partial products are calculated and the right hand vector is placed into the appropriate high order or low order array before these inner products and the corresponding right hand vector element are summed (see Wilkinson p84.).

Let $\mathrm{e}]$ be the correction vector and $\mathrm{r}]$ the residuals of the right hand side:

We now solve $A[\mathrm{e}[]=\mathrm{r}[\mathrm{f}$ for $\mathrm{e}[\mathrm{by}$ means of the $L / U$ matrices.

An error value,xerr, is calculated by xerr=norm_correction vector/norm_x vector.

Iteration terminates when:

1) the norm of the residual vector is $<=0.0$

2) the sum of the norm_ $x$ vector plus the norm_correction vector

$=$ norm_ $\mathrm{x}$ vector(i.e., no change within the range of the significant digits of the given computer.).

3) the number of iterations is $>=3$.

Note: The norm_x vector $=\operatorname{Max}|x[i]|$, for $i=1$ to $n$.

In the above DBX run the correction_vector $=$ dxnrm $=4.14483262526724646 \mathrm{e}-14$ after 2 iterations. Thus, for the Sun workstation, condition 2) is satisfied.

Verification is complete for this test data.

Test will be made on other data 0 input. 


\section{T. Kishi December 2, 1992}

It should be noted by forcing itrefn.f to be executed, subroutines stpp.f, srtsum.f, and sortr8 are also exercised (see DBX runs on these subroutines using the test data).

This is a test problem listed in Numerical Mathematics and computing by Ward Cheney David Kincaid ${ }^{2}$

The answers for $x$ 's agree (i.e., compare the results printed in the ITREFN.DOC against results on pages 203 and 204 of sec. 6.1 in the above text book).

\section{November 25, 1992 T. Kishi} $* \$$

With respect to "ill-conditioned" matrices, the code incorporates tests for linear dependence (i.e., call to the subroutine lindep.f) in order to eliminate the occurrence of obviously ill-formed matrices as input data. EQ3/6 uses logarithmic iteration variables in order to reduce the disparities in the order of magnitudes of the elements of the matrix, and sets bounds for the number of iterations. Other techniques that are used in aiding convergence are described in Section 9 of the EQ3NR document. Thus, the question of "ill-conditioned" matrices is addressed outside the procedures of sgefa.d, sgeco.f, sgesl.f, and itrefn.f. In fact, EQ3NR and EQ6 in the process of normal NewtonRaphson iteration do under certain conditions drive the matrix to singularity. This condition is trapped and diagnosed. In EQ6, for example, it may signify the disappearance of a mineral!

\section{5) EQPTBL.DOC (Table 1)}

This document is the list of software modules tested and verified for EQPT. The entries are; name, type, and brief summary of the module.

A great deal of emphasis is placed on calculating the "norm" (in our case, the reciprocal of the "condition number $1,2,3,10,16,24,25$ labeled rnorm in $\mathrm{EQ} 3 / 6$ ), but it appears to be a necessary and not a sufficient condition for convergence to a solution. The value rnorm is not used extensively in $\mathrm{EQ} 3 / 6$.

At this time, the verification of EQPT, together with modules accessed in EQLIB, is complete with no programming errors found in this package. The above and other documents are noted in Section 3.3.

\subsection{EQ3NR}

Both DBX and FrameMaker software tools have been used on the set of EQ3NR codes. While all of the EQ3NR I/O subroutines have been reviewed, the main focus has been on The Newton-Raphson procedure. This procedure consists two parts: pre-Newton-Raphson optimization and hybrid New- 
ton-Raphson iteration. The detailed analysis began with arrset.f (see Diagram 2 or eq3list.tre) up to the call to newton.f for the pre-Newton-Raphson and newton.f for the latter.

\subsubsection{Pre-Newton-Raphson}

Unit testing and verification of this phase of EQ3NR pointed out some discrepancies in the programming and in the EQ3NR document. Most of the errors are in the document.

In the calculation of the Davies Equation, the procedure for $\log \gamma_{i}$ in gdavie.f differs from equation (85) in the EQ3NR document.

For gdavie.f:

$$
\operatorname{glgc}(i)=\log \gamma_{i}=\frac{z^{2}}{2}\left(-2 A_{\gamma_{1}, 10}\left(\frac{\sqrt{I}}{(1+\sqrt{I})}-0.2 I\right)\right) .
$$

In $\mathrm{EQ} 3 / 6$ code, the variable $z s q 2$ is defined as $z s q 2=\frac{z_{i}^{2}}{2}$. Thus, a compensating factor of 2 must appear in the above equation (i.e., -2 as in $-2 A_{\gamma, 10}$ ) in order to match the Davies Equation.

Equation (85) in the EQ3NR document:

$$
\log \gamma_{i}=-A_{\gamma, 10^{2}}{ }^{2}\left(\frac{\sqrt{l}}{(1+\sqrt{l})}+0.2 I\right) \text {. }
$$

The difference is the sign of the last term and should be "." as in gdavie.f.

There is also another error in Section 3.2 of the EQ3NR document. In equation (86), the number "2" in the last term multiplying the parenthesized value (0.2) should omitted.

The correct equation is:

$$
\text { actlwc }=\log a_{w}=\left(-\frac{\sum^{m}}{2.303}+\frac{2}{3} A_{\gamma, 10^{1 / 2}}\left(\sigma(\sqrt{I})-0.2 A_{\gamma, 10^{2}} I^{2}\right) \Omega .\right.
$$

Two problems occurred in the calculation of Pitzer's Equations. The first is a quality assurance problem and will be resolved by providing a copy of the reference ${ }^{11}$ to the Record Center. The function $\mathrm{J}(\mathrm{x})$ and its derivatives approximated by a Chebyshev polynomial suggested by Harvie and Weare (1980) are cited in the EQ3NR document but equations are only available in Harvie's Ph.D. thesis. Luckily, a copy of the paper was found from which the procedure in hjdd.f could be verified. The second is a document error. The equation (103) in Section 3.5.1 is incorrect for two reasons: 1) the ionic strength I is missing as a factor in the second term of the second line in equation (103); 2) the factor $\frac{1}{\Omega}$ should be $-\frac{1}{\Omega}$ (i.e., the minus value). The correct equation is as follows:

$$
\begin{gathered}
\quad l n a_{w}=-\frac{\sum_{\Omega}^{m}}{\Omega}-\frac{1}{\Omega}(I f(I)-f(I)) \\
+\left(-\frac{1}{\Omega}\right)\left(\sum_{i j}\left(\lambda_{i j}(I)+I \lambda_{i j}^{\prime}(I) m_{i} m_{j}+2 \sum_{i j k} \mu_{i j k} m_{i} m_{j} m_{k}\right) .\right.
\end{gathered}
$$

In verifying subroutine gcscal.f, equation (99) in the EQ3NR document is incorrect. The correct equation should be: 


$$
\log \gamma_{i}^{(2)}=\log \gamma_{i}^{(1)}+\frac{z_{i}}{z_{j}}\left(\log \gamma_{j}^{(2)}-\log \gamma_{j}^{(1)}\right) \text {. }
$$

The information for above can be found in the documents: GBDOT.DOC, GCOEFF.DOC, GDAVIES.DOC, GDLGXW.DOC, and PITZER.DOC.

\section{1) GBDOT.DOC}

This document lists the source code gbdot.f and the mathematical equations involved in the "bdot" and other models that calculate activity coefficients of aqueous species. The corrections necessary in the EQ3NR document are shown above. This module calls gcscal.f (see comments above).

\section{2) GCOEFF.DOC}

This document contains the test and verification comments for gcoeff.f. This module is simply a driver routine that calls gbdot.f, gdavie.f, nbsgam.f, and gcscal.f.

\section{3) GDAVIES.DOC}

This document lists the equations used in the Davies Model for activity coefficients. The discrepancies between the code and the EQ3NR document are also listed (see comments above for gdavie.f).

\section{4) GDLGXW.DOC}

This document lists the procedure for calculating $\frac{\partial \log x_{w}}{\partial \log m_{s}}$.

$$
\frac{\partial \log x_{w}}{\partial \log m_{s^{\prime}}}=\frac{-\frac{x_{w}}{\Omega}\left(m_{s^{\prime}}-\sum_{r}^{r_{T}} \frac{b_{s^{\prime} r} m_{s^{\prime \prime}}}{b_{s^{\prime \prime} r}}\right)}{\left(1-\frac{x_{w}}{\Omega} \sum_{r}^{r_{T}} \frac{b_{w r_{r}} m_{s^{*}}}{b_{s^{\prime \prime} r}}\right)} \quad s^{\prime} \neq w, s_{B}
$$

\section{5) PITZER.DOC}

This document lists the "reverse engineering" of Pitzer's equations starting from gcoeff.f and subsequent calls to hjdd.f, elmdd.f, gelam.f, gesum.f, gselm.f, fdd.f, gslam.f, gdd.f, gssum.f gmsum.f, gsdsm.f, gmdsm.f, but not necessarily in this sequence. There is an error in equation (103) (Section 3.5.1.) as noted above.

The next phase is defined in Section 9.6 of the EQ3NR document. In this section of the computation, Arrset.f loops through a procedure consisting of passes and cycles until rough convergence criteria are satisfied. Within this loop, arrset.f calls arrsim.f that calculates starting estimates then calls msolvr.f for rough convergence.This is followed by calls to ncmpx.f (calculates log activities and $\log$ molal concentrations), betas.f (computes "beta" residual functions), and ngcadv.f (calculates ionic strengths, activity coefficient-gcoeff.f, and calls ncmpx.f). The subroutines msolvr.f, ncmpx.f, ngcadv.f, and betas.f are all called in the Newton-Raphson iteration (NR) but they have been already verified. All of the modules and sub calls have been examined and verified and no discrepancy has been found. 
Documents relevant to this part are BETASFNS.DOC, NCMPX.DOC, NGCADV2.DOC, GCOEFF.DOC from above, and MSOLVR.DOC from EQPT verification. The document PRENRTBL.DOC summarizes the modules called in this part of EQ3NR.

\section{1) BETASFNS.DOC}

This document lists the "reverse engineering" of the equations in betas.f that compute the NewtonRaphson residual functions $\beta$ and $\alpha$.

\section{2) NCMPX.DOC}

This document lists the "reverse engineering" of the computations in ncmpx.f necessary to write the matrix from the zvclg1 array, $z$.

\section{3) NGCADV2.DOC}

This document lists the "reverse engineering" of the computations in ngcadv. $f$ that recalculates the activity coefficients of aqueous species, and then recalculates the concentrations of dependent aqueous species. This module calls gsigm.f, gxi.f, and gshm.f.

4) PRENRTBL.DOC (Table 2)

This document summarizes the modules called in this part of EQ3NR and is Table 2.

\subsubsection{Newton-Raphson Method}

The following has been taken from the file EQ3NR.tre ******

$$
\text { showing the NEWTON-RAPHSON loop }
$$

616:1

$* * * * * 200$

**Test for convergence
617:I

618:I

619:I

620:1

621:1

622:1

623:I

624:I

625:I

626:I

627:1

628:1

629:1

*******
+NEWTON --.- +-@BETAE continue 
The routines above not already reviewed are: newton.f, calls other modules; nrstep.f, initiates one Newton-Raphson iteration; and matrxe.f, called by nrstep.f, calculates the Jacobian matrix.

The subroutine matrix.f, referenced as matrxe in the call chain, was determined to be in error. The error was found when the calculations of the Jacobian in an earlier write-up did not agree with the official document. Programming changes were necessary in balcon.f, matrix.f, and non-procedural changes made in bkdeq3.f and eq3nr.f. Since these were Software Quality Affecting, a new set was obtained from the Configuration Management (CM). The differences in the source codes are listed in an output titled $3 R 127$ src.sdf,3/19/93, Wolery ${ }^{30}$. The corrected version is EQ3/6 Version 7.2a, January 12, 1994.

At the same time, other document errors were also identified.

These are:

1) a missing " ." sign in the equation (292)

$$
\begin{gathered}
J_{s s_{B}}=-2.303 \sum_{r=1}^{r_{T}} \frac{u_{s^{n} s} m_{s^{n} b_{s_{B} r}}}{b_{s^{n} r}} \\
J_{s s_{B}}=-2.303 \sum_{r=1}^{r_{T}} b_{s_{B} r} H_{s r}
\end{gathered}
$$

2) a change in "." to " + " sign in equation (293)

$$
\begin{gathered}
J_{s s^{\prime}}=2.303\left(u_{s^{\prime} s} m_{s^{\prime}}-\sum_{r=1}^{r_{T}} \frac{u_{s^{\prime} s^{\prime}} m_{s^{\prime \prime}} b_{s^{\prime} r}}{b_{s^{\prime \prime} r}}\right) \\
J_{s s^{\prime}}=2.303\left(u_{s^{\prime} s^{\prime}} m_{s^{\prime}}-\sum_{r=1}^{r_{T}} b_{s^{\prime} r} H_{s r}\right)
\end{gathered}
$$

3) a change in "-" to "f" sign in equation (298)

$$
J_{s s_{B}}=-2.303 \sum_{r=1}^{r_{T}} b_{s_{B}} H_{z r}
$$

4) a change in "-" to " + " sign in equation (299)

$$
J_{s s^{\prime}}=2.303\left(z_{s^{\prime}} m_{s^{\prime}}-\sum_{r=1}^{r_{T}} b_{s^{\prime} r} H_{z r}\right) ;
$$

5) a missing equation after equation (341)

$$
J_{s s}=-1.0
$$


In addition to the documents listed above (i.e., BETASFNS.DOC, NCMPX.DOC,MSOLVR.DOC, and NGCADV2.DOC), the document MATRIX.F.DOC shows the updated equations. NEWTON.DOC and NRSTEP.DOC are included as references. The document EQ3NRTBL.DOC summarizes the subroutines called in the Newton-Raphson loop.

1) MATRIX.F.DOC This document references the subroutine matrix.f that calculates the elements of the Jacobian Matrix $J_{s s^{\prime}}$. The corrected equations are listed.

\section{2) EQ3NRTBL.DOC (Table 3)}

This document is the summary of the modules in the Newton-Raphson calculation.

\subsubsection{Post Newton-Raphson Procedures}

The modules for the post Newton-Raphson procedures have been examined and verified. The main routine is scripx.f that computes values for the EQ3NR output file. The subroutine dsiplx.f is listed but not used.

The relevant documents are SCRIPX.DOC, HPSAT.DOC, and POSTNRTBL.DOC, which summaries the subroutines in this set.

\section{1) HPSAT.DOC}

This document lists the "reverse engineered" calculations for the most stable composition of a given solid solution.

\section{2) SCRIPX.DOC}

This document lists the few equations used in the output phase of EQ3NR.

3) POSTNRTBL.DOC (Table 4)

This document is the summary of the modules in the Post-Newton-Raphson phase.

The verification of EQ3NR is completed.

\subsection{EQ6}

The focus on EQ6 verification effort has been on the computational section of the codes that begins after the read-in of input files. This starts with a call to ncmpz.f, which computes all parameters necessary to write the Jacobian matrix from the zvclg1 array. "Reverse Engineering" is the method of verification. The "Software Requirements Document" or its equivalent can be found partly in the 
EQ6 manuals, partly in the EQ3NR manual, and sometimes in previous EQ3/6 write-ups. From the Flint EQ6.tre (Diagram 3), the verification process separated naturally into three parts: 1) ncmpz.f . to eqcalc.f, 2) eqcalc.f to path.f, and 3) path.f to end of EQ6 run. As in the case of verifying EQ3NR, once a module is verified, its procedure is accepted as verified in any further call. This is also true for modules already verified for EQPT and EQ3NR.

There were three discrepancies found in EQ6. The first occurs with respect to subroutine akmatr.f. The coding for the module is correct but the EQ6 document has an error in the recursion formula EQ6 (156):

$$
D_{m, n}=D_{m, n-1} w_{n}+D_{m-1, n-1} \times m \quad, m=2, i-2, n=m+1, i \text {, where the factor } \mathbf{m} \text { was missing }
$$

The second is an error in the code in the subroutine hpsat.f. While this subroutine was reviewed during the V \& V of EQ3NR, the error only occurs in a calculation that seldom, if ever, is executed. Namely, the error would arise only when non-ideal solution model is called on (See HPSAT.DOC for error). The correction will be in EQ3/6 Version 7.2b.

The third error appears in the lambda.f subroutine. When the Third-Order Maclaurin Model for the Binary Solution (Site-Mixing Parameters) is selected, a local variable "a" is not initialized prior to equation (EQ3NR (188)): $\operatorname{lamlg}(2, n x)=f a c *\left(a+b * z x 2+c * z x^{*} z x 2\right)$. Actually, the value of " $a=\left(W_{1 \psi}+W_{2 \psi} / 2+W_{3 \psi} / 6\right)$ " is set equal to zero in wterm.f in order to satisfy a symmetry condition (See EQ3NR Document Section 4.2). The fix, a=0, will be in $E Q 3 / 6$ Version 7.2b.

There is one other comment that should be noted with respect to the subroutine akmatr.f. This subroutine is purported to fill the $D_{m, n}$ (akm matrix), but the diagonal elements are initialized elsewhere-in eq6.f. The need to initialize the diagonal elements elsewhere should be, at least, noted in the subroutine preamble. This is done in EQ3/6 Version $7.2 \mathrm{~b}$.

All of the EQ6 documents are listed in Table 5 and in Appendix B. Those that are relevant to the statements above are: AKMATR.doc, HPSAT.doc, and LAMBDA.doc.

All of the modules in the 3 parts listed above for EQ6 have been reviewed and verified. All of the other modules that appear in the EQ6.tre listing deal with I/O or initialization of arrays. These are generally referenced when searching for a variable that appears as left member of an equation (i.e., where a variable is SET-see EQ6.xrf file).

\subsection{Comments on the Verification Process}

\subsection{Meeting Requirements}

Since the EQ3/6 Software Package was written prior to the existence of SQAP ${ }^{17}$ and QP 3.2, Rev. 2 , the applicable requirements for test and verification are described in Section 6.3 of SQAP and corresponding parts of new QP 3.2, Rev. 2. The software requirements were delineated from the four EQ3/6, Version 7 documents (UCRL-MA-110661 PT I - IV), as Version 7.1 was treated as "acquired code." However, it has been necessary to review other technical papers cited in the above set in order to complete this assignment. In particular, the EQPT document is a user's manual for 
this data base package but does clearly specify the functionality of fitting thermodynamic data. It uses standard subroutines from a well established mathematical library called LINPACK in order to preprocess data for $E Q 3 / 6$. In the absence of a criteria for accepting a well tested public domain software package, such as LINPACK, each module used by EQPT (i.e., EQ3/6) was reviewed and verified.

\subsection{Applying the Software Test and Verification Plan (STVP ${ }^{23}$ )}

The procedures described in the STVP ${ }^{23}$ (Appendix, ISP-NF-07, Revision 1) have been applied to the EQ3/6 suite of codes with one exception, "Reverse Engineering (See 2.1.4)."

The use of the UNIX software tool DBX has been one of trial-and-error. The use of a script file for DBX command sequence does help in reducing the number of typing errors and provides a convenient way of documenting the event. However, the output can be overwhelming when the arrays are large. The output files from various runs of DBX may be edited files (see 3.3 Deliverables). The "*.outx" files consist of the DBX script file, source listing, and test and verification comments. Since these are ASCI files, each file could be used as the DBX script file providing the particular source code line numbers match and the input file corresponds to the DBX run.

For EQ3NR and EQ6, the verification process has been one of proof reading the documents as well as verifying the programs. In the case of discrepancy between document and code, it needs to be determined whether the code or the document, or both are in error. While the software tool DBX was used extensively for EQPT, the method of "Reverse Engineering" has served its purpose well for verifying EQ3 and EQ6 calculations.

Each line of code has a use in the EQ3/6 suite of codes. Each branch of a conditional statement was examined and each branch followed during the "Walkthrough" process. The author of the code has set the lower threshold of a real number to less than 100esps, where eps is particular computer's smallest real number. Also, the magnitude for log of a real number is tested and set to less than 999 . Thus, the possibility of a "crash," as a result of a fault condition (i.e., operand overflow and/or operand underflow) set during a procedure, is minimized if not avoided.

When a software error was determined to be actually or potentially quality affecting, the error was recorded in the electronic notebook, the TL and the code author were notified, a new source code was obtained from the CM system, and the process of reverification continued from this point.

Each call-tree (i.e., EQPT.tre, EQ3NR.tre, and EQ6.tre produced by using FLINT) was used as a guide in determining the sequence of verifying the subroutines and functions. The order of the modules listed in each Summary Matrix (See Section 3.3.1) corresponds very closely to each respective call-tree. The equation numbers listed in column 3 of each Summary Matrix correspond to the equation numbers in their respective documents (i.e., References 27, 28, and 29).

\subsection{Deliverables}

The Deliverables consist of: 


\subsubsection{Electronic Notebook}

ZNOTEBK.DOC-Framemaker document - Appendix A.

\subsubsection{DBX files}

*.outx-ASCII file - list of files in Appendix C.

These files are too large to be included in this report and will be available at the Records Information System (RIS).

\subsection{3 "Reverse Engineered" files}

*.DOC or *.doc-Framemaker document - list of files in Appendix B.

These files are too large to be included in this report and will be available at the Records Information System (RIS).

\subsubsection{Flint output files}

“*.xrf" and "“*.tre" from FLINT for EQPT, EQ3NR, and EQ6.

Note: EQPT.tre (Diagram 1), EQ3NR.tre (Diagram 2), and EQ6.tre (Diagram 3) are not included in this report and are available at the Records Information System (RIS) in a document named Tables and Diagrams. The names of the cross-reference files produced by FLINT (i.e., *.xrf files) are listed in Appendix D. Since the files are too large to be included in this report, they have been sent to the Records Information System (RIS).

The data files, output files, and the source codes for EQPT, EQ3NR, and EQ6 used for test and verification are not part of the deliverables of this STVR.

\subsubsection{Summary Matrix}

The following documents consist of three column tables summarizing the modules that consist mainly of mathematical (short procedure) or numerical calculations. The exception is EQPT which includes system calls and character manipulating procedures. These tables are not included in this report and will be available at the Records Information System (RIS) in a document called Tables and Diagrams.

\section{1) EQPTBL.DOC (See Table 1).}

This table summarizes the subroutines that interface with the operating system, read and check input data, initialize arrays in EQPT. The subroutines are listed in the sequence as they appear in the *.tre output from FLINT. 


\section{2) PRENRTBL.DOC/ Pre-Newton-Raphson (See Table 2)}

This table summarizes the subroutines that interface with the operating system, read and check input data, initialize arrays including the initial Jacobian matrix for the Newton-Raphson iteration. The subroutines are listed in the sequence as they appear in the *.tre output from FLINT. Repeated references to a given subroutine present in the *.tre output may appear only once in this summary table. This allows the reviewer to keep relevant information close at hand rather than flipping through an alphabetized table of subroutines. The author has purposely left out the subroutines starting with timdat.f and up to, but not including, arrset.f. These routines, while they have been reviewed in detail, are basically calls to system services and FORTRAN input/output $(I / O)$ calls in order to process formatted data. The mathematical and numerical (usually simple arithmetic calculation) procedures start with arrset.f.

\section{3) Newton-Raphson (See Table 3)}

This table summarizes the subroutines that are called by subroutine newton.f. The subroutine msolvr.f and its associated subroutines are summarized in a separate document. This sequence of programs is the heart of the eq $3 \mathrm{nr}$ procedure.

4) POSTNR.DOC/Post Newton-Raphson (See Table 4)

The modules in this set edit the results of the Newton-Raphson iteration. When the NR iteration fails, the subroutine ndiag.f checks and prints various parameters for diagnostic purpose.

5) EQ6 (See Table 5)

EQ6 summary matrix lists subroutines after input files have been read-in and various arrays have been initialized. This sequence begins with a call to ncmpz.f at line eq6.f:1470. 


\subsection{References}

1. Carnahan, B., H. A. Luther, and Wilkes, Applied Numerical Methods, John Wiley and Sons, New York, 1969. (NNA. 901005.0043)

2. W. Cheney, D. Kincaid, Numerical Mathematics of Computing, 1985, Chapter 6.

3. Cline, A. K., Moler, C. B., Stewart, G. W., and Wilkinson, J. H., An estimate for the Condition Number of a Matrix, SIAM J. Numer. Anal., 16 (1979), pp. 368-375.

4. Daveler, S. A., and Wolery, T. J., 1991, EQPT, A Data File Preprocessor for the EQ3/6 Software Package: User's Guide and Related Documentation (Version 7): UCRL-MA110662-PT-II, Lawrence Livermore National Laboratory, Livermore, California. (NNA. 930129.0101)

5. DBX, Source Level Debugger: UNIX operating system utility.

6. Dongarra, J. J., Bunch, J. R., Moler, C. B., Stewart, G. W., LINPACK Users' Guide, SIAM, (1979). (NNA. 891106.0200)

7 Elden, L., Linde Wittmeyer-Koch, Numerical Analysis, San Diego, CA, Academic Press, 1990.

8. FLINT, FORTRAN-Lint, Fortran Source Code Analyzer: Information Processing Techniques Corporation (IPT)

9. FRAMEMAKER, Frame Technology Corp., 1010 Rincon Circle, San Jose, CA, 95131.

10. Hager, W. W. (1984), "Condition Estimates," SIAM J. Sci. Stat. Comput., 5, pp. 311 316.

11. Harvie, C. E., 1981, Theoretical Investigations in Geochemistry and Atom Surface Scattering: Ph.D. thesis, University of California, San Diego.

12. Harvie, C.E., and Weare, J. H., 1980, The Prediction of 'mineral solubilities in natural waters, Geochimica et Cosmochimica Acta, v. 44, pp. 981-997. (NNA. 921023.002)

13. IEEE Standard for Software Verification and Validation Plans, ANSI/IEEE Std 10121986.

14. INTERA Environmental Consultants, Inc., 1983, Geochemical Models Suitable for Performance Assessment of Nuclear Waste Storage: Comparison of PHREEQE and EQ3/6: ONWI-473, Battelle Memorial Institute, Office of Nuclear Waste Isolation, Columbus, Ohio. 
15. ISP-NF-07, Revision 1, Individual Software Plan for Initial Qualification of EQ3/6, November 25, 1992. (NNA. 921023.0026)

16. Johnston, R. L., Numerical Methods, N. Y., J. Wiley \& Sons, 1982.

17. LLNL Yucca Mountain Project Software Quality Assurance Plan (SQAP), Rev 0, December 14, 1989. (NNA. 900302.0042)

18. LLNL YMP Software Configuration Management System Technical Implementing Procedure, TIP-YM-11 Rev. 0, 18 March 1991.

19. Maron, M. J., Numerical Analysis, Macmillan Publishing Co., (1987).

20. Nordstrom, D. K., Geochemical Thermodynamics, Menlo Park, Benjamin/Cummings Pub., 1985.

21. Puigdomenech, I., and Bruno, J., 1988, Modelling uranium solubilities in aqueous solutions: Validation of a thermodynamic data base for the EQ3/6 geochemical codes: SKB Technical Report 88-21, Swedish Nuclear Fuel and Waste Management Company, Stockholm, Sweden. (NNA: 890425.0186)

22. QP 3.2, Revision 2, Software Quality Assurance Procedure, June 21, 1994.

23. STVP, Appendix, ISP-NF-07, Revision 1, Individual Software Plan for Initial Qualification of EQ3/6, November 25, 1992.

24. D. S.,Watkins, Fundamentals of Matrix Computations, John Wiley \& Sons, 1991,Chapter 2.

25. J. W., Wilkinson, The Solution of Ill-conditioned Linear Equations, in A. Ralston \& H. S. Wilf, Mathematical Methods for Digital Computers, vol. 2, John Wiley and Sons, pp. 65-93.

26. Wolery, T. J., 1991a, EQ3/6, A Software Package for Geochemical Modeling of Aqueous Systems: Package Overview and Installation Guide (Version 7): UCRL-MA110662-PT-I, Lawrence Livermore National Laboratory, Livermore, California.

27. Wolery, T. J., 1991b, EQ3NR, A Computer Program for Geochemical Aqueous Speciation-Solubility Calculations: Theoretical Manual, User's Guide, and Related Documentation (Version 7): UCRL-MA-110662-PT-III, Lawrence Livermore National Laboratory, Livermore, California. (NNA. 921218.0010)

28. Wolery, T. J., 1991, EQ6, A Computer Program for Reaction-Path Modeling of Aqueous Geochemical Systems: Theoretical Manual, User's Guide, and Related Documentation (Version 7): UCRL-MA-110662-PT-IV, Lawrence Livermore National Laboratory, Livermore, California. (NNA. 930127.0028)

29 Wolery, T. J., Calculation of Chemical Equilibrium Between Aqueous Solution and Minerals: The EQ3/6 Software Package, UCRL-52658, (1979). (RA) 
30. Wolery, T. J., EQ3NR source code differences ( $L L N L$ internal document), 3R127src.sdf, $3 / 19 / 93$. 


\section{Appendix A}

\section{ZNOTEBK.DOC}

**** Establish an electronic notebook --TTP-YM-12,5/27/92 ****

Date entered Mon Aug 31 10:45:41 PDT 1992 Tadashi Kishi

Entry $1 * * * *$

Received 2 floppies containing the source files EQ3_6version 7.1 on 8/28/92 10:00am from

Tom Wolery. The files are compressed $Z$ format

stvp.txt

171 cd eq3_6_7v1

$174 \operatorname{tar} \mathrm{xvpf} / \mathrm{dev} / \mathrm{rfd} 0 \mathrm{c}$

176 efd

$179 \operatorname{tar} \mathrm{xvpf} / \mathrm{dev} / \mathrm{rfd0c}$

220 efd

224 mkdir eqpt

225 mkdir eqlib

226 mkdir eq3

227 mkdir eq6

228 uncompress *.Z

$230 \mathrm{~cd} \mathrm{eqpt}$

231 in /us/kishi/eq3_6_7v1/eqptR81.fsc

237 lw5 INSTALL.tst

238 hi > log.aug

Fri Aug 28 14:26:07 PDT 1992

Date entered March 19, 1993 8:45 PDT Tadashi Kishi

Entry $2 * * * *$

* \$run matrx3.in in order to localize possible error

$* \$$ in matrix.f

* \$the above dumps values for the evaluation of J_ss' in matrxe.f

$* \$ 3 / 15 / 93$ TW is checking on equation (341)/9.8.11

* $\$$ earlier write-up shows a difference in the calculation of the Jacobian J_ss'

* $\$$ On $3 / 7 / 93$, Tom Wolery stated that the calculation in question was incorrect.

* He said that he had changed the calculation on an earlier version, but it

* appears the changes in the code were done incorrectly or not at all.

* $\$$ On $3 / 19 / 93$, Tom Wolery has related to me on changes that will be made

* \$and he will provide a complete set of the $\mathrm{EQ} 3 / 6$ package. There are also

* $\$$ corrections in the documents that must be made.

* 3/19/93 10:48 AM PDT Tad Kishi

*\$The programming changes are in balcon.f, matrix.f, and non-procedural changes in 
*\$bkdeq3.f and eq3nr.f.

*\$On June 23, 1993, I sent a memo to Bill Glassley and copies to Bill Halsey, Tom Wolery, * \$and Nick Krissa (acting group leader in place of Teri Quinn) reporting a probable error

*\$ in eq3NR Pitzer's equation. The error may be an error in the doculment(EQ3NR-UCRL *\$MA-110662 PT III) equation (103) on page 45.

August 11, 1993

Tom Wolery transferred EQ3.6, Version 7.2 on two floppies.

January 7, 1994

Tom Wolery transferred EQ3/6 Version 7.2a on two floppies.

Verification of EQ6 will proceed with this version.

April 15, 1994

The following was sent on April 15, 1994 to Bill Glassley and Tom Wolery:

I have been reviewing the subroutine akmatr.f and I have found a discrepancy between the coding of the matrix $\underline{D}_{m, n}$ and the equation (156) in the EQ6 document (See attached pages). I have also reread the document UCRL 52658 and B. Carnahan,... Applied Numerical Method in an attempt to resolve the discrepancy. I have found that the values of the $\underline{D}_{m, n}$ in the UCRL report is consistent with equation (156) in the official EQ6 document Version 7.0. The multiplying factor krow is in question.

Please advise me

April 18, 1994

The discrepancy has been resolved as follows:

1. The coding of akmatr.f is correct as it stands providing the user understands that diagonal elements of the matrix is initialized elsewhere to $n$ !. Preferably, the initialization of the diagonal to $\mathrm{n}$ ! should be imbedded in the code as a one time procedure since the preamble states, "this routine computes the akm matrix ...."

2. The EQ6 document must be corrected in the last paragraph on page 204:

The matrix $\underline{D}$ being upper triangular, all the diagonal elements are $n$ ! (i.e., $i, j=n$ ) and all elements in the lower triangle are zero. The following recursions permit calculation of the off-diagonal elements in the upper triangle. For the first row, we have that:

$$
D_{1, n}=D_{1, n-1} w_{n}
$$

where $\mathrm{n}$ goes from 2 to $\mathrm{i}$. The remaining elements may be calculated from the following recursion formula:

3. The equation EQ (156) on page 205 should be corrected to multiply the last term by $\mathrm{m}$ :

$$
J_{m, n}=D_{m, n-1} w_{n}+D_{m-1, n-1} \times m \quad, m=2, i-2, n=m+1, \text { l }
$$

4. It should be also noted that UCRL 52658 is in error with respect to the matrix D. 
May 17, 1994

There is an error in the subroutine hpsat.f that deals with non-ideal solid solutions as follows:

286: $\mathrm{j}=1$

287:c nn \# components present, check to see if xbarh is present,

288:c if not, increment index

289: do $26 \mathrm{i}=1$,nn

********Ioop 44 will not exit if conditional on line hpsat.f:290 fails ************

290: 44 if (nend(i,nx).ne.0 .and.

291: * itracx(i).eq.0) go to 45

292: $\quad \mathrm{j}=\mathrm{j}+1$

293: go to 44

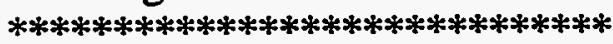

294: $45 \operatorname{xbar}(\mathrm{j}, \mathrm{nx})=\operatorname{xbarh}(\mathrm{i})$

295: if (xbar(j,nx).lt.0.) write (noutpl, *)'xbar(',j,nx,' <0'

296:c log mole fraction

297: $\quad x \operatorname{bar} \lg (\mathrm{j}, \mathrm{nx})=\operatorname{tlg}(\operatorname{xbarh}(\mathrm{i}))$

298: $j=j+1$

299: 26 continue

There is also a discrepancy in the description of the variable jsol in the EQ3NR Appendix:

Note:

jsol Array whose values define chosen models of activity coefficients in solid solutions. Dimensioning: jsol(nxtpar). Usage: jsol(nx) is the flag for the $n x-$ th solid solution:

$=0 \cdot$ Ideal solution

(The data files currently support no other options.)

Yet in the subroutine hpsat.f line 224, jsol(nx) is tested against the value 1:

224: if(jsol(nx).eq.1) go to 1000

One of the input routine sets jsol(nx) $=1$ for all $n x$ 's. Obviously then, the non-ideal solid solution model is never exercised.

However, in order to be consistent with EQ6, the Appendix of EQ3NR must reflect this change in the value of jsol.

Tom Wolery has stated that he will appropriately address the modeling of the non-ideal solid solution at a later time.

June 1, 1994

While examining the subroutine lambda.f, I have found that a local variable " $a$ " is not initialized when The Third-Order Maclaurin Model for the Binary Solution (Site-Mixing Parameters) is selected. This error is probably a simple oversight since the calculation involving the interaction coefficients that define " $a$ " result in $a=0$ (See wterm). This procedure in wterm.f satisfies a certain condition for the Third-Order Maclaurin Model.

August 17, 1994

All of the minor bugs found in EQ3/6 Version 7.2a have been fixed and will be designated as

EQ3/6 Version 7.2b (See letter to Dr. Ardyth M. Simmons dated August 17, 1994). 
September 14, 1994

The following items were found in the EQ3NR and EQ6 documents during the V \&V activities but these are not "quality" affecting.

"oops" items from EQ6 document UCRL-MA-110662 PT IV

1. page 6: see Wolery et al. (1990) - is not listed in References.

2. page 19: EQ3/6 6 - should be EQ3/6 ?

3. page 28: (See Van Zeggeren and Storey, 1970) is not listed in References.

4. page 22: EQ3NR Theoretical Manual and User's Guide (Wolery, 1992b)

information in Reference section is in error:

UCRL-MA-110662-PT-IV should be UCRL-MA-110662-PT-III.

5. page 47: format error "data" is in Greek letters.

September 14, 1994

The V \& V activity for the EQ3/6 suite of codes has been completed and the Software Test and Verification Report (STVR) has also been completed as of this date.

The deliverables for this V \& V are being assembled for delivery to the Records Information System (RIS).

Tadashi Kishi 9/14/94

"Work performed under the auspices of the U.S. Department of Energy by Lawrence Livermore National Laboratory under Contract W-7405-ENG-48. 


\section{Appendix B}

The following are Framemaker 4.0 documents (Some names may be appended with .FM4). These files are not included in this report. The names in bold weight are EQ6 documents and the names in plain weight are EQ3NR documents. Some of the documents that are shared by both EQ3NR and EQ6 are set in italics.

AFFUNC.DOC

AKMATR.DOC

BETASFNS.DOC

BETAZ.DOC

DERIV.DOC

DERSPC.DOC

EQ6.DOC

EQCALC.DOC

ESCALC.DOC

FDOMSP.DOC

GAUSS.DOC

GBDOT.DOC

GBFAC.DOC

GCOEFF.DOC

GDAVIES.DOC

GDLGXW.DOC

GSIGM.DOC

HPSAT.DOC

INTEGR.DOC

ITREFN.DOC 
JGIBBS.DOC

LAMBDA.DOC

MATRIX.DOC

MATRIX.F.DOC

MODEXZ.DOC

MSHIFT.DOC

MSOLVR.DOC (same as SIMSOLVR.DOC)

MTRXSLV.DOC

NCMPX.DOC

NCMPZ.DOC

NCMPZ2.DOC

NEWTON.DOC

NGCADV.DOC

NGCADV2.DOC

NRSTEP.DOC

OPTMZR.DOC

PABSSW.DOC

PATH.DOC

PHSDRP.DOC

PITZER.DOC

RAFF.DOC

RCOND.DOC (was called GAUSS.DOC)

RDERIV.DOC

REACTS.DOC

RFDIFF.DOC 
RSATCH.DOC

RTAYLR.DOC

RTCALC.DOC

SATCHK.DOC

SCRIPX.DOC

SEARCH.DOC

SFNCAF.DOC

SFNCPD.DOC

SFNCPM.DOC

SFNCRE.DOC

SFNCRI.DOC

SFNCRR.DOC

SGEFA.DOC

SHFTZ.DOC

SIMSOLVR.DOC

SSFUNC.DOC

SSHIFT.DOC

TAYLOR.DOC

TAYLR2.DOC

TSTEP.DOC

ZFDIFF.DOC 


\section{Appendix C}

\section{DBX ASCII files.}

Each of the following files consists of two or three separate files concatenated together as one file.

These files not included with this report, are:

The first is a source file input to DBX

The second is the output file from the DBX run using the source file.

The third is a comment file for a special DBX run (files with **).

betas.outx

dawfix.outx

elim.outx

gbfac.outx

gcoeff.outx

gcoeffNZ.outx **

gcoeffpit.outx

gcoeffpit2.outx

gcoeffpit3.outx

gcoeffpit4.outx

gcsts.outx

itrefn.outx $* *$

lindep.outx

lindepx.outx

matrix.outx

matrx3.outx **

msolvr.outx ***

msolvr.outxtd ** 
ncmp.outx *

NCMPNZ.outx

ncmp $2 x N Z$.outx **

ngcadv1.outx

ngcadv2.outx

nrstep2.outx

nrstep288.outx

The following are EQPT *.outx files

chump.outx **

flpars.outx **

funcy.outx **

getlu.outx **

gnenb.outx ***

isamax.outx **

ofiles.outx **

openin.outx **

openou.1.outx **

openou.outx $* *$

pcraq.outx ***

pdpz2.outx **

polx.outx **

prendi.outx **

rdbdot.outx **

rdpar.outx **

rdwele.outx ** 
rdwttl.outx **

rscal.outx $* *$

sasum.outx $* *$

saxpy.outx **

scal.outx $* *$

sdot.outx **

Sgeco.outx

sgeco.135.outx

sgeco.180.outx

sgeco.199.outx

sgeco.217.outx

sgefa.1.outx (see SGEFA.outx)

sgefa.x.outx (see SGEFA.outx)

SGEFA.outx **

sgesl.outx **

sgesl.outx6.1**

sgesl.outx6.1b**

sort8.outx **

srtsum.outx $* *$

sscal.outx **

stripl.outx **

timdat.outx **

undfiw.outx **

WRPAR.outx *** 


\section{Appendix D}

The following are FLINT *.xrf files that have been edited in order to reduce the size of the output. Only those variables with the keyword "Set" are retained in the *.xrf files. The three files, not included with this report, are:

1. eqpt.xrf

2. eq3NR.xIf

3. eq6.xrf 


\section{Appendix E}

\section{Acronyms}

ASCII

$\mathrm{CM}$

DBX

EISPACK

EQLIB

EQPT

FLINT

I/O

ISP

LINPACK

L/U

NF

QP3.2

RIS

SPARC

SQAP

STVP

STVR

SUN

TL

UNIX

V \& V
Name of a standard set of characters for computer usage

Configuration Management (Defined in QP3.2)

Program debugging system on UNIX platform

See LINPACK

Library of subroutines for EQ3/6 (many from LINPACK)

$\mathrm{EQ} 3 / 6$ data base program

Software tool for FORTRAN programs (output: errors, call tree, cross-references)

Input and/or Output

Individual Software Plan

Library of mathematical subroutines in the public domain (solving linear equations)

Decomposition of a matrix into Lower and Upper Triangular matrices

Near Field (Yucca Mountain Project)

Quality Procedure 3.2 - Software Quality Assurance

Records Information System

Name of class of computers built by SUN

Software Quality Assurance Plan superceded by QP3.2.

Software Test and Verification Plan

Software Test and Verification Report

Name of workstation manufacturer (SUN Microsystem)

Task Leader

Name of operating system

(Software) Verification and Validation 
